\title{
External Quantum Efficiency Improvement with Luminescent Downshifting Layers: Experimental and Modelling
}

\author{
H. Ahmed, ${ }^{1}$ S. J. McCormack, ${ }^{1}$ and J. Doran ${ }^{2}$ \\ ${ }^{1}$ School of Engineering, Trinity College Dublin, Dublin, Ireland \\ ${ }^{2}$ Dublin Energy Lab, Dublin Institute of Technology, Dublin, Ireland \\ Correspondence should be addressed to H. Ahmed; hahmed@tcd.ie
}

Received 7 October 2015; Accepted 15 December 2015

Academic Editor: Akihide Wada

Copyright (C) 2016 H. Ahmed et al. This is an open access article distributed under the Creative Commons Attribution License, which permits unrestricted use, distribution, and reproduction in any medium, provided the original work is properly cited.

Core-shell quantum dots $\mathrm{CdSe} / \mathrm{ZnS}$ and lumogen yellow organic dye are characterized by their inclusion in luminescent downshifting (LDS) layers. Layers were deposited on top of crystalline silicon cell (c-Si), dye synthesized solar cell (DSSC), and cadmium telluride (CdTe) minimodules. External quantum efficiency measurements for the solar cell/LDS devices are discussed. Experimental results were compared with an optical model developed by Rothemund, 2014.

\section{Introduction}

Luminescent downshifting (LDS) is an optical approach to increase a solar cell's spectral response by using luminescent materials to convert high energy photons to lower energy before the interaction with the solar cells occurs [1-7]. The downshifted photons have wavelength which may better match the photosensitivity spectral response of the solar cell as illustrated in Figure 1. A typical LDS consists of luminescent species such as organic dyes and quantum dots doped in a transparent polymer sheet applied on top of PV cells [8-13].

\section{Experimental}

2.1. LDS Layers and Devices Fabrication. The luminescent materials used in this investigation were core-shell quantum dots CdSe/ZnS purchased from Cytodiagnostics, Canada, and lumogen yellow dye developed and manufactured by BASF, Germany. A clear polymer poly(methyl methacrylate) (PMMA) (Carl Roth GmbH + Co.KG) was used for the encapsulation of LDS layers.

The LDS layers were prepared as follows.

The QDs/dye were mixed in toluene and sonicated in an ultrasonic bath for 10 minutes. The mixture was then added to PMMA solution (50 wt\%), magnetically stirred for 20 minutes, and then placed in an ultrasonic bath for another
15 minutes. Prepared solutions were drop cast on glass substrates and cured for 72 hours at $25^{\circ} \mathrm{C}$ under a vacuum of $800 \mathrm{mbar}$. Uniform layers were obtained when removed from the glass substrate. The average thickness of the layers was measured by white light interferometer technique and found to be $0.95 \pm 0.05 \mu \mathrm{m}$.

The luminescent quantum yield (LQY) measurements of LDS films were measured using the integrating sphere method $[14,15]$ found to be $0.58 \pm 0.08$ for QD-LDS layer and $0.87 \pm 0.08$ for dye-LDS layer.

UV/Vis/NIR absorption spectroscopy was used to measure the absorption characteristics of the LDS layers investigated in this study. The UV/Vis/NIR absorption spectrometer used was a Perkin Elmer Lambda 900. The emission spectra were measured by optically pumping samples using a monochromated light source using a luminescence spectrometer (Perkin Elmer LS55B). QD and dye absorption and emission spectra in a PMMA film (0.09 wt\% and $0.9 \mathrm{wt} \%)$ are shown in Figures 2 and 3, respectively.

Monocrystalline silicon cells $(2 \times 2 \mathrm{~cm}$, Sunrydz, Germany), dye-sensitized solar cells $(1 \times 1 \mathrm{~cm}$, Solar Print, Ireland, the details fabrication of which are confidential), and CdTe minimodules $(15.24 \times 15.24 \mathrm{~cm}$, Advanced Solar Power INC, China) were used for assessing the downshifting effect of the LDS layers. Devices were fabricated by directly 


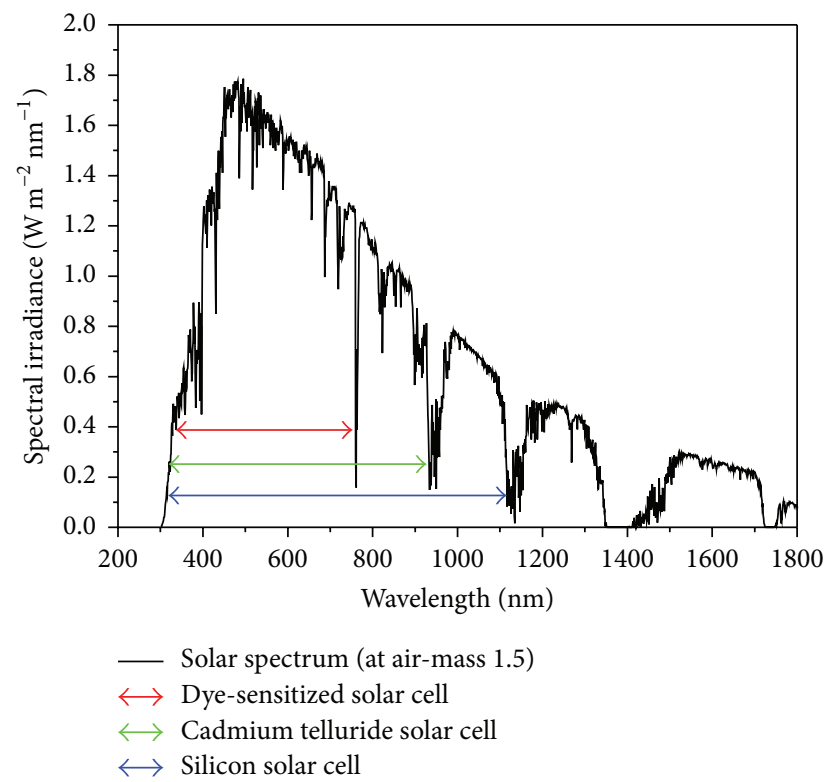

FIGURE 1: Solar spectrum and PV cells typical absorption ranges.

depositing the LDS layers on top of the solar cells surface, using PMMA solution to glue the layers. The deposited layers were left for three days to dry and stick to the cells.

2.2. External Quantum Efficiency Measurements. External quantum efficiency (EQE) measurement is an indicator of how well the solar cell converts incident photons (of a specific wavelength) to electricity. The EQE is the ratio of the number of charge carriers that are collected by the solar cell to the number of photons of a given wavelength entered into the solar cell [16].

The EQE system used was Bentham PVE300. The system uses a monochromatic probe source from a TMc300, $300 \mathrm{~mm}$ focal length monochromator, and a dual Xenon/quartz halogen light source providing optimum illumination from 300 to $1100 \mathrm{~nm}$. The monochromatic beam area is $1.85 \mathrm{~mm}^{2}$ and is directed at the centre of the cell, measuring the photocurrent generated by the cell at each wavelength. A calibrated silicon detector $(10 \times 10 \mathrm{~mm}$ with calibration traceable to national standards) was used to calibrate the system for the measurements of spectral responsivity by determining the power in the probe beam as a function of wavelength, with uncertainties of $5 \%$ for $300-400 \mathrm{~nm}$. The PVE300 is entirely automated through a computer based interface using the Benwin+ software program. The probe source is operated in the AC mode to discriminate the photocurrent generated by the probe from that generated by the DC bias of one sun $\left(1000 \mathrm{w} / \mathrm{m}^{2}\right)$ during the measurements. To obtain the AC signal, an optical chopper is used at frequency of $600 \mathrm{~Hz}$ with a lock-in amplifier having a 474 transformer that passes only the optically chopped signal to be amplified. However, in the case of DSSC cells, which have a particularly slow electron transport, it is necessary to either run the monochromatic probe beam in DC mode or use slower chopping frequencies, less than $10 \mathrm{~Hz}$. The DSSC output is passed through a 497

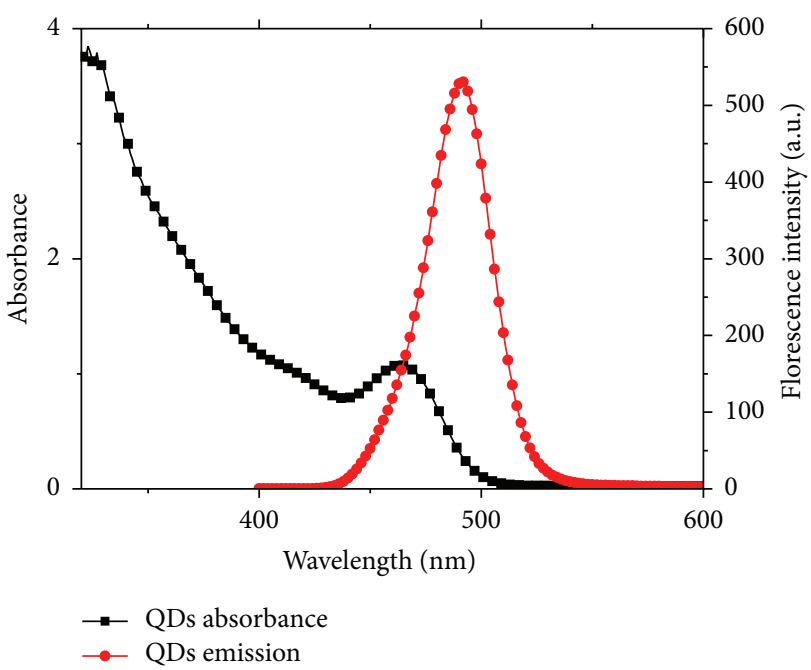

Figure 2: Absorbance and fluorescence emission spectra of CdSe/ZnS QDs.

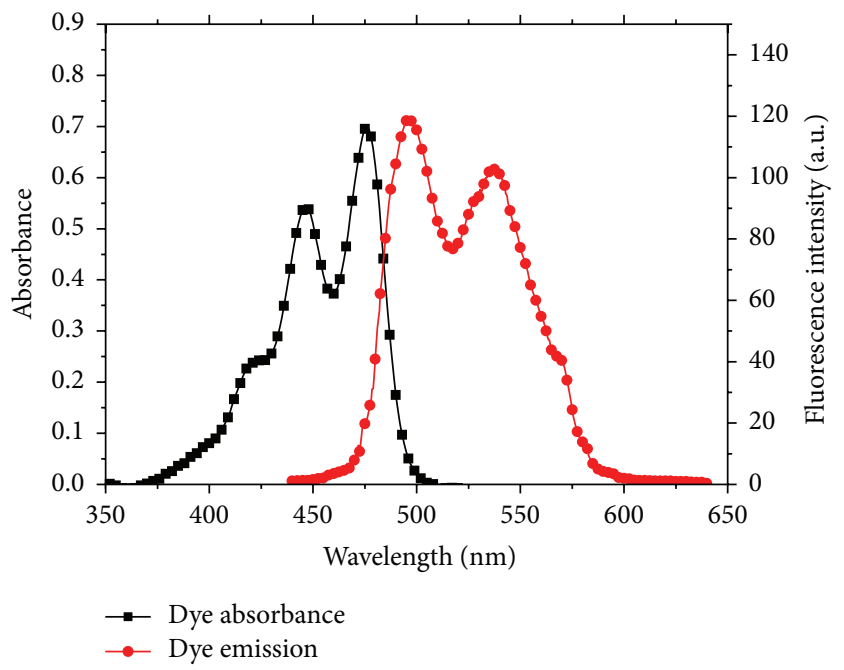

FIGURE 3: Absorbance and fluorescence emission spectra of lumogen yellow dye.

transimpedance amplifier prior to being passed to the lockin amplifier.

\section{Analyzing EQE Spectra Using Rothemund Optical Model}

To evaluate the effect of the LDS layer on the EQE of the solar cells investigated in this paper, the model developed by Rothemund, 2014, was used [17]. The aim of this analysis was to validate the experimental results obtained and to gain an insight into LDS processes. This particular model was chosen because it is simple to implement and it has shown an excellent agreement with simulated EQE and measured EQE spectra in a number of studies $[5,8,18,19]$. In this model, the incoming photon flux density was separated into two fractions, the absorbed fraction $\left(\mathrm{Abs}_{f}\right)$ and the transmitted 


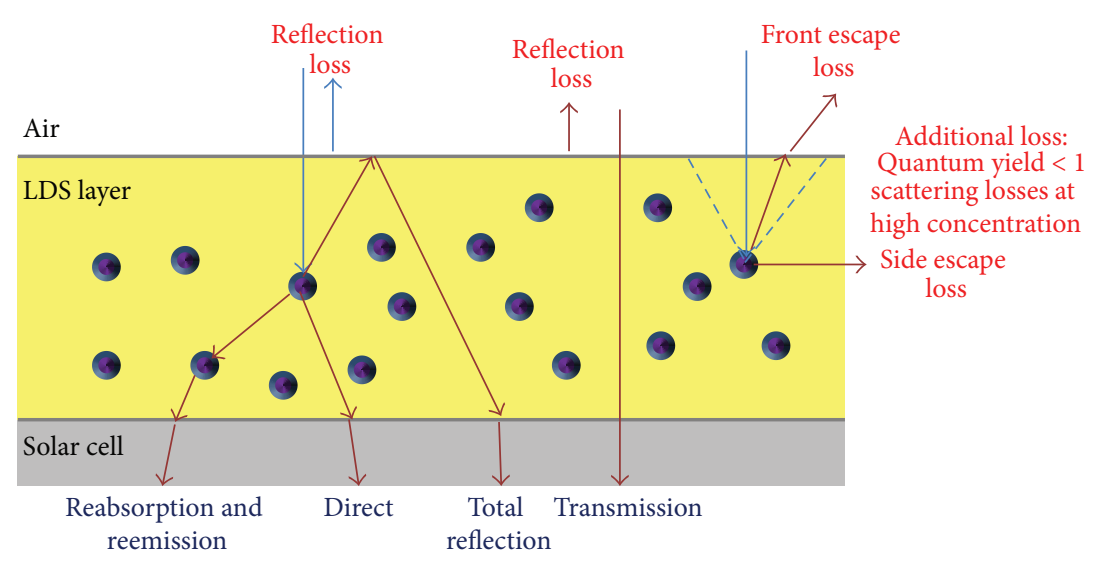

FIGURE 4: Optical losses in LDS layers (reproduced from [17]).

fraction ( $\operatorname{Trans}_{f}$ ). Abs $s_{f}$ is the fraction of light that is absorbed and reemitted by the luminescent particles while Trans ${ }_{f}$ is the fraction of light that reaches the solar cell as if no LDS layer was attached.

The number of photons absorbed by the LDS layer compared to the incoming photons flux can be calculated for each wavelength using the Lambert-Beer law as follows:

$$
\operatorname{Abs}_{f}(\lambda)=\frac{\varphi_{\mathrm{Abs}}}{\varphi_{\text {incoming }}}=1-\exp \left(-\Lambda_{\mathrm{Abs}} \cdot A(\lambda)\right) .
$$

The term $\Lambda_{\mathrm{Abs}}$ is a scaling factor which describes the absorption $A(\lambda)$ and it is directly proportional to the concentration of the luminescent species and the LDS layer thickness. follows:

The number of photons transmitted can be calculated as

$$
\operatorname{Trans}_{f}(\lambda)=1-\operatorname{Abs}_{f}(\lambda) .
$$

The effect of the absorbed fraction of light $\operatorname{Abs}_{f}\left(\lambda, \Lambda_{\mathrm{Abs}}\right)$ that has been downshifted to longer wavelength is described by introducing the LDS efficiency $\eta_{\text {LDS }}$ term. LDS $\eta_{\text {LDS }}$ is the fraction of the photons that is downshifted and reaches the solar cell to that absorbed by the LDS layer. All the loss mechanisms (illustrated in Figure 4) due to the LDS layer such as imperfect photoluminescent quantum yield, escape cone loss, and self-absorption are considered by the parameter $\eta_{\text {LDS }}$. Therefore, $\eta_{\text {LDS }}$ can be estimated as follows:

$$
\eta_{\mathrm{LDS}}=\eta_{\mathrm{PL}}\left(1-L_{\mathrm{esc}}\right)\left(1-L_{\mathrm{reAbs}}\right)\left(1-L_{\mathrm{other}}\right) \text {. }
$$

$\eta_{\mathrm{PL}}$ is the photoluminescent quantum yield of the LDS layer, $L_{\text {esc }}$ is front-side escape loss due to isotropic emission, which is calculated to be $12.5 \%$ [4], $L_{\text {reAbs }}$ accounts for the self-absorption affect, and $L_{\text {other }}$ accounts for any other loss such as reflectance and escape through the side of the LDS layer. The reflectance due to the incorporation of the LDS materials in the matrix was considered to be zero for simplicity and any effect due to reflectance is considered in the term $L_{\text {other }}$ of (3).

For an efficient LDS layer, absorbed photons must be reemitted at wavelengths where the EQE of the solar cell is high so they can be readily converted into electricity. Therefore, emission spectral matching (ESM), which is defined as the fraction of the emission that overlaps with the EQE $[5,17]$, is used in the model to calculate LDS efficiency. ESM is given as follows:

$$
\operatorname{ESM}=\frac{\int \operatorname{PL}\left(\lambda^{\prime}\right) \mathrm{EQE}_{\mathrm{ref}}\left(\lambda^{\prime}\right) d \lambda^{\prime}}{\operatorname{PL}\left(\lambda^{\prime}\right) d \lambda^{\prime}} .
$$

EQE of the LDS layer is derived by considering the photons absorbed by the LDS layer and those transmitted and is given as follows:

$$
\begin{aligned}
\mathrm{EQE}_{\mathrm{LDS}}= & \mathrm{Abs}_{f}(\lambda) \cdot \eta_{\mathrm{LDS}} \cdot \mathrm{ESM}+\operatorname{Trans}_{f}(\lambda) \\
& \cdot \mathrm{EQE}_{\mathrm{ref}}(\lambda) .
\end{aligned}
$$

This model needs only the emission and the absorption of the LDS layer plus the EQE of the solar cell as an input. EQE of the LDS layer is fully described by the LDS layer efficiency, $\eta_{\mathrm{LDS}}$, and $\Lambda_{\mathrm{Abs}}$, the absorbance scaling factor.

\section{Results and Discussion}

4.1. QD-LDS Layers on Top of c-Si Solar Cell. Figure 5 presents the experimental EQE measured for $\mathrm{c}-\mathrm{Si}$ solar cells with and without QD-LDS layers together with the best fit for $\mathrm{EQE}_{\mathrm{LDS}}$ using (5) and varying only the LDS layer efficiency, $\eta_{\text {LDS }}$, and $\Lambda_{\text {Abs }}$, the absorbance scaling factor. An excellent agreement is obtained of the simulated spectra for QD-LDS layer and its experimental data. EQE of the bare c-Si solar cell has shown a very poor optical response and hence low EQE at wavelengths below $400 \mathrm{~nm}$. At these wavelengths, EQE is enhanced for the cell encapsulated with QD-LDS layer, reaching 23\% at $300 \mathrm{~nm}$ and up to $28 \%$ at $365 \mathrm{~nm}$. The improvement in EQE occurs because photons are absorbed by the QDs molecules and then reemitted at longer emission wavelengths. ESM calculated for the Si solar cell was 0.90. Both absorbance scaling factor, $\Lambda_{\mathrm{Abs}}$, and LDS efficiency, $\eta_{\mathrm{LDS}}$, were varied and the best fit was obtained at values of 0.5 and 0.46 , respectively. The LDS layer efficiency value is considered reasonable in the light of LQY measured for QD-LDS layer in PMMA 


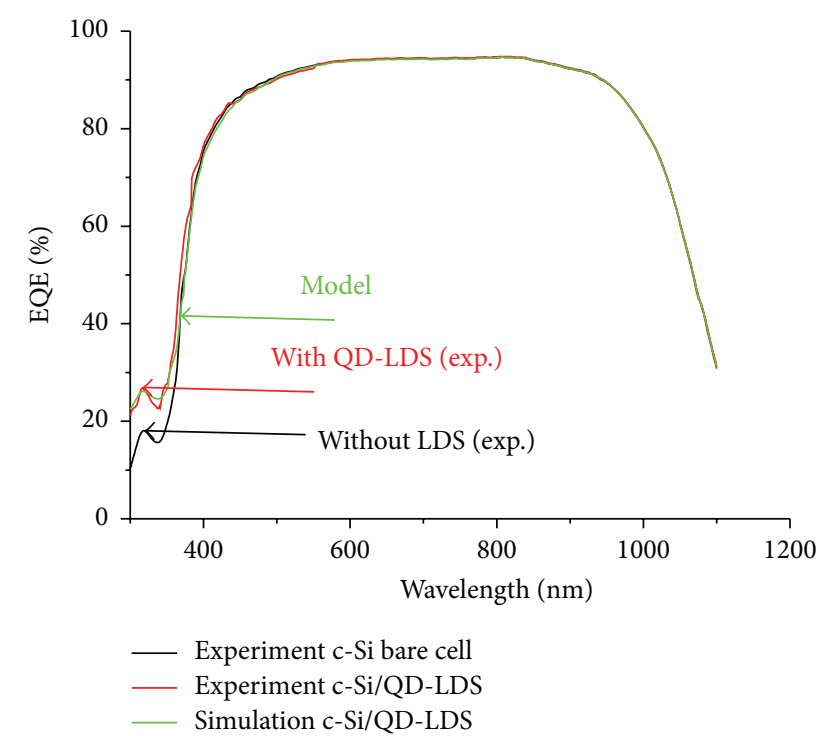

FIGURE 5: Experimental EQE spectra of c-Si solar cells with QD-LDS layer and spectra derived using the optical model of Rothemund, 2014 [17].

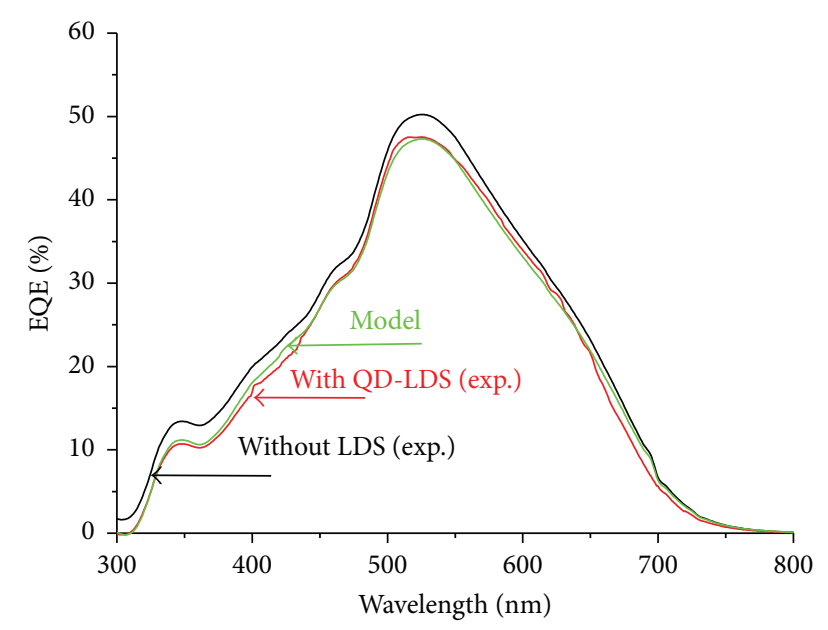

Experiment DSSC bare cell
_ Experiment DSSC/QD-LDS
Simulation DSSC/QD-LDS

FIGURE 6: Experimental EQE spectra of DSSC solar cells with QD-LDS layer and spectra derived using the optical model of Rothemund, 2014 [17].

which is $0.58 \pm 0.08$. Considering all the loss mechanisms in (3), escape loss alone is reducing the layer efficiency to 0.51 $(0.58$ (1-Lesc $(0.125)=0.5075)$ and the other $5 \%$ is attributed to reabsorption loss and any other possible loss due to the presence of LDS layer. For QDs with high LQY such as reported by De Mello Donegá et al., 2003, 85\% [20], the efficiency of LDS layer using (3) would be around $69 \%$.

4.2. QD-LDS Layers on Top of DSSC Solar Cells. Figure 6 presents the experimental EQE measured for DSSC solar cells with and without QD-LDS layer together with the best fit for $\mathrm{EQE}_{\mathrm{LDS}}$ using (5). An excellent agreement was obtained

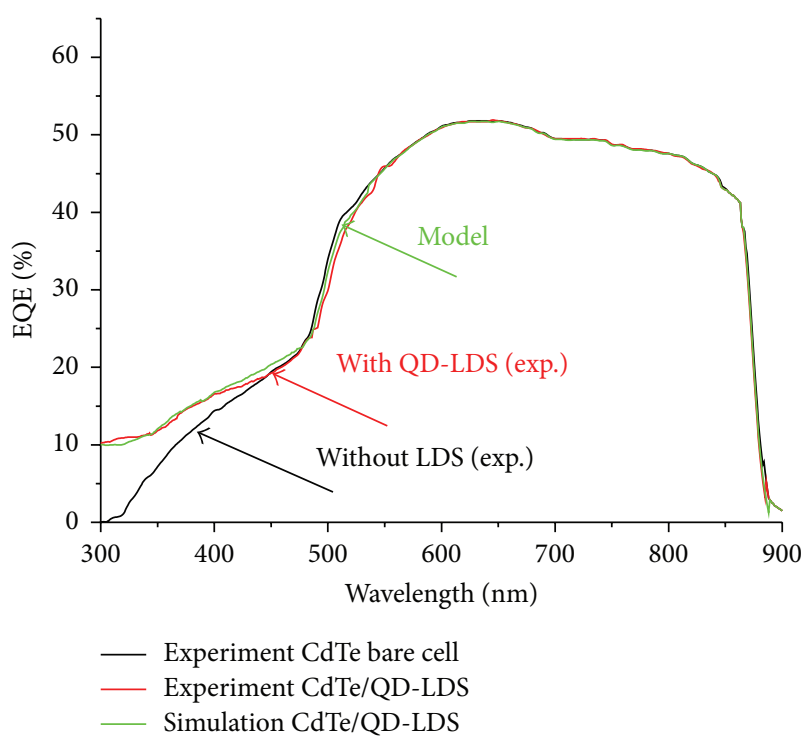

FIGURE 7: Experimental EQE spectra of CdTe solar cells with QDLDS layer and spectra derived using the optical model of Rothemund, 2014 [17].

between simulated spectra for QD-LDS layer and experimental data by varying only the LDS layer efficiency $\eta_{\text {LDS }}$ while fixing the absorbance scaling factor $\Lambda_{\text {Abs }}$ to the same values obtained in modelling LDS layer on top of c-Si solar cell which was 0.5. Absorbance scaling factor is dependent on the concentration and the thickness of the LDS layer which has been assumed to be the same for the QD-LDS layer. EQE for the DSSC solar cell has shown overall decrease of about $\sim 3 \%$ in EQE for QD-LDS layer. We were previously shown [13] that this decrease arising due to optical losses because of the specific DSSC geometry in which the $3 \mathrm{~mm}$ thick cover glass significantly decreases the fraction of the emitted photons transmitted to the photoactive layer in the DSSC. ESM calculated for the DSSC solar cell was 0.39. The LDS layer efficiency $\eta_{\text {LDS }}$ best fit was for a value of 0.42 . This is lower than what has been calculated for c-Si solar cell which was 0.46 . This could be due to effect of reflection at the interface (DSSC top cover is glass and c-Si cell has an ARC coating on the top cell) which might increase escape loss in DSSC solar cell.

4.3. QD-LDS Layers on Top of CdTe Minimodules. Figure 7 presents the experimental EQE measured for CdTe mini modules with and without QD-LDS layers together with the best fit for $\mathrm{EQE}_{\mathrm{LDS}}$ using (5) and varying only the LDS layer efficiency, $\eta_{\mathrm{LDS}}$, while fixing $\Lambda_{\mathrm{Abs}}$ the absorbance scaling factor to a value of 0.5. An excellent agreement was obtained of simulated spectra for QD-LDS and its experimental data. The EQE of the CdTe minimodules with QD-LDS layer shows an improvement in EQE, reaching 10-16\% within 300$485 \mathrm{~nm}$. There is a slight reduction at $485-505 \mathrm{~nm}$ due to the overlap between the absorption and emission spectra which causes self-absorption. This effect is due to the relative difference in EQE between the QDs absorption and emission wavelengths which is not great enough to overcome the loss 
TABLE 1: The absorbance scaling factor and the LDS layers efficiency of QD-LDS layer with the EQE enhancements calculated for c-Si, DSSC solar cells, and CdTe minimodules.

\begin{tabular}{lccc}
\hline & $c-S i$ & DSSC & CdTe \\
\hline$\Lambda_{\text {Abs }}$ & 0.5 & 0.5 & 0.5 \\
$\eta_{\text {LDS }}$ Q QD-LDS & 0.46 & 0.42 & 0.44 \\
EQE enhancement $\%$ & $23-28$ & -3 & $10-16$ \\
& $(300-365 \mathrm{~nm})$ & & $(300-485 \mathrm{~nm})$ \\
\hline
\end{tabular}

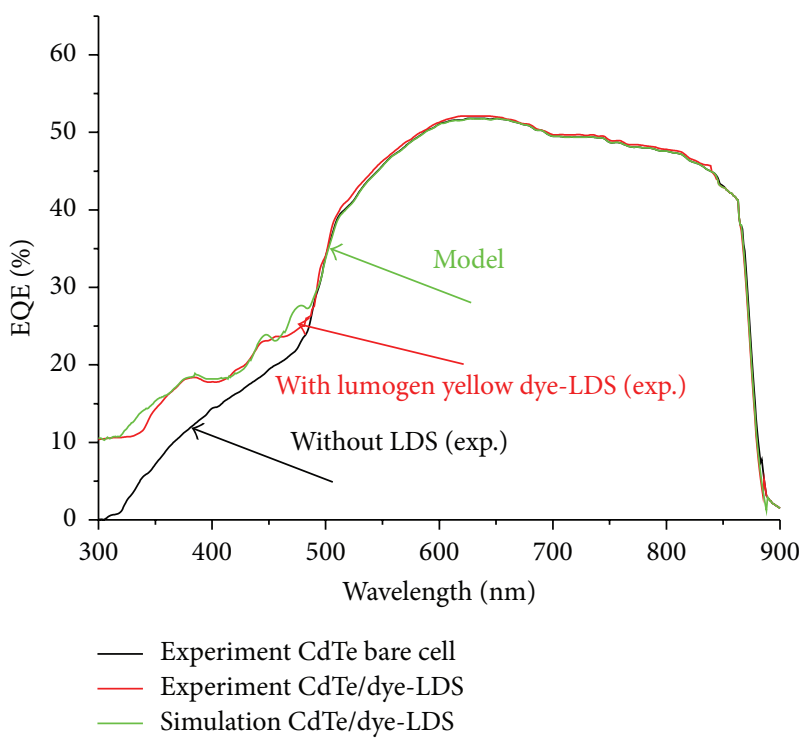

Figure 8: Experimental EQE spectra of CdTe solar cells with lumogen yellow dye-LDS layer and spectra derived using the optical model of Rothemund, 2014 [17].

associated with reabsorption in these regions [21]. ESM calculated for the CdTe solar cell was 0.31. LDS layer efficiency $\eta_{\text {LDS }}$ best fit was for a value of 0.44

The absorbance scaling factor, $\Lambda_{\mathrm{Abs}}$, and the LDS layer efficiency, $\eta_{\mathrm{LDS}}$, for QD layer are presented in Table 1 together with the EQE enhancement calculated for each cell.

\subsection{Lumogen Yellow LDS Layers on Top of CdTe Minimodules.} Figure 8 presents the experimental EQE measured for CdTe minimodules with and without LDS layers of lumogen yellow dye together with the best fit for $\mathrm{EQE}_{\mathrm{LDS}}$ using (5) and varying only the LDS layer efficiency, $\eta_{\mathrm{LDS}}$, and $\Lambda_{\mathrm{Abs}}$, the absorbance scaling factor. An excellent agreement was obtained of simulated spectra for lumogen yellow dye-LDS layer and its experimental data. EQE has increased significantly reaching 10-30\% within 300-485 $\mathrm{nm}$, compared with QD-LDS layers in Figure 5. ESM calculated for the CdTe minimodules was 0.4 . Absorbance scaling factor, $\Lambda_{\mathrm{Abs}}$, and efficiency, $\eta_{\mathrm{LDS}}$, of the LDS layer were varied and the best fit was obtained at value of 0.7 for both. LQY obtained for lumogen yellow dyeLDS layer was $87 \%$. Considering all the loss mechanisms in (3), escape loss alone is reducing the lumogen yellow LDS efficiency to $76 \%(87 \%(1-\operatorname{Lesc}(0.125)=0.7613)$ and the other

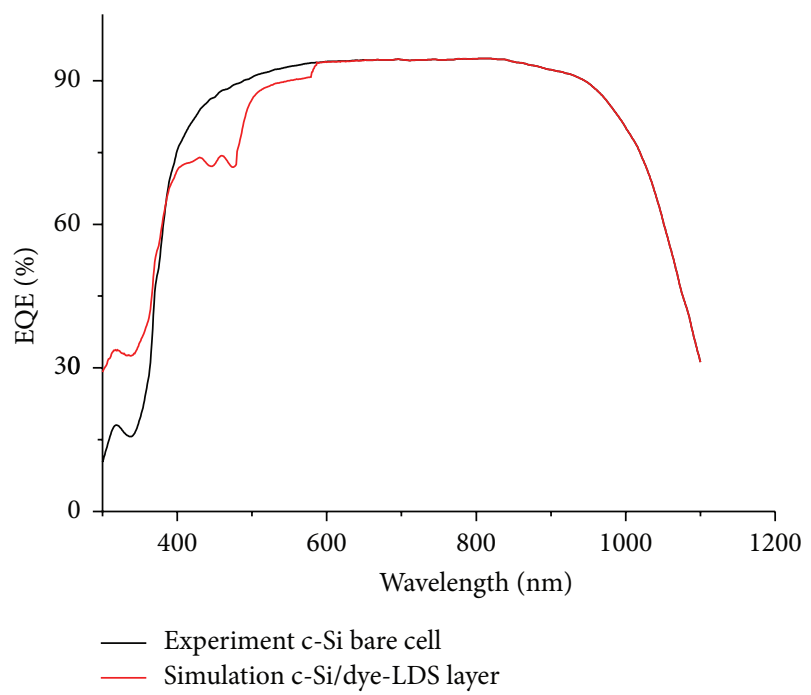

FIGURE 9: Experimental EQE spectra of c-Si solar cell with simulation of lumogen yellow dye-LDS layer with $\eta_{\text {LDS }}$ of 0.7 using the optical model of Rothemund, 2014 [17].

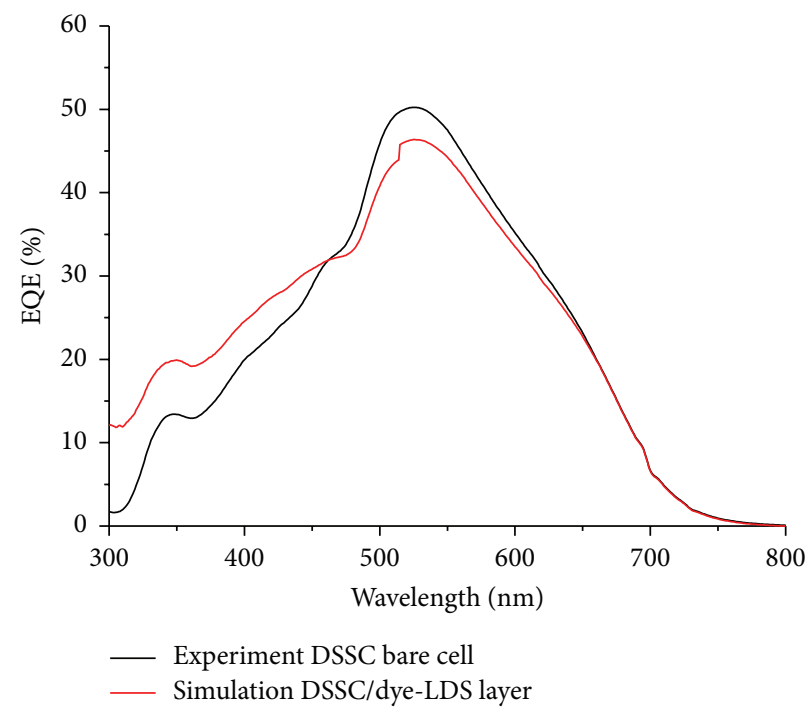

FIGURE 10: Experimental EQE spectra of DSSC solar cells with simulation of lumogen yellow LDS with $\eta_{\text {LDS }}$ of 0.7 using the optical model of Rothemund, 2014 [17].

$6 \%$ is attributed to reabsorption loss and any other possible loss due to the presence of dye-LDS layer.

The input parameters used for modelling lumogen yellow dye-LDS layers on the CdTe minimodules were used to estimate the performance of the lumogen yellow LDS on top of c-Si and DSSC solar cells. The simulation results are shown in Figures 9 and 10, respectively. EQE of the bare c-Si solar cell with lumogen yellow dye-LDS layer has shown a better optical response compared to QD-LDS layer in Figure 5 reaching (30 to 40\%) at wavelengths less than $400 \mathrm{~nm}$. However, at wavelengths between 400 and $580 \mathrm{~nm}$, EQE was found to be decreased and most probably this decrease is due to self-absorption of lumogen yellow dye. 
TABLE 2: The absorbance scaling factor and the LDS layers efficiency of dye-LDS layer with the EQE enhancements calculated for CdTe minimodules.

\begin{tabular}{lccc}
\hline & $c-S i$ & DSSC & CdTe \\
\hline$\Lambda_{\text {Abs }}$ & 0.7 & 0.7 & 0.7 \\
$\eta_{\text {LDS }}:$ dye-LDS & 0.70 & 0.70 & 0.70 \\
EQE & $30-40$ & $12-32$ & $10-30$ \\
enhancement \% & $(300-365 \mathrm{~nm})$ & $(300-450 \mathrm{~nm})$ & $(300-485 \mathrm{~nm})$ \\
\hline
\end{tabular}

EQE of the bare DSSC solar cells with lumogen yellow dye-LDS layer has shown a significant increase reaching $12-32 \%$ within $300-450 \mathrm{~nm}$ compared to the QD-LDS in Figure 6 which has shown an overall decrease in EQE. Some decrease in EQE within 460-600 nm was observed for lumogen yellow dye-LDS layer due to self-absorption. Absorbance scaling factor and LDS layer efficiency for lumogen yellow dye layers on CdTe minimodules are summarised in Table 2. The EQE enhancement calculated for solar cell is presented too.

The initial value used for LDS efficiency, $\eta_{\text {LDS }}$, as an input in the model was estimated from the LQY value obtained for the layer minus the optical loss expected $(\sim 15 \%)$ due to the presence of LDS layer. The absorbance scaling factor, $\Lambda_{\text {Abs }}$, and $\eta_{\text {LDS }}$ were then both varied until the best fit was obtained. The absorbance scaling factor was fixed, assuming that the concentration of the layer and the thickness were the same, since both were optimised. The variation observed in $\eta_{\text {LDS }}$ for QD-LDS layer, for example, $0.46,0.42$, and 0.41 for C-Si, DSSC, and CdTe, respectively, could be attributed to experimental errors while fabricating the layers or in the deposition process of LDS layer on top of the solar cells. Moreover, it could be due to optical losses due to different cell technologies. The difference in $\Lambda_{\mathrm{Abs}}$ for QD-LDS and dyeLDS layers is clearly due to the difference in concentration in each layer. LDS efficiency was higher for lumogen yellow LDS layer because the LQY was higher (>87\% compared to 58\%).

\section{Conclusion}

This paper investigated the performance of c-Si, DSSC solar cells, and CdTe minimodules encapsulated by PMMA solid sheet doped with quantum dots $\mathrm{CdSe} / \mathrm{ZnS}$ and lumogen yellow dye. External quantum efficiency measurements have shown that an increase in the short-wavelength response of the $\mathrm{c}-\mathrm{Si}$ and $\mathrm{CdTe}$ minimodules was achieved due to the presence of QD-LDS and dye-LDS layers. DSSC solar cell shows an overall decrease in EQE for QD-LDS layer; it is believed to be due to optical losses because of the specific DSSC geometry. The optical model of Rothemund, 2014 [17], was found to be an excellent match with EQE measured for the fabricated devices. LDS efficiency $\eta_{\text {LDS }}$ extracted from the model for QD-LDS layer was $44 \pm 2 \%$ while for lumogen yellow LDS was 70\%.

\section{Conflict of Interests}

The authors declare that there is no conflict of interests regarding the publication of this paper.

\section{Acknowledgments}

The authors would like to acknowledge funding from the Irish Higher Education Authority under the Strand 3 Program (SEAR Project) and the European Research Council (PEDAL Project no. 639760). The authors also acknowledge the FOCAS Institute for use of equipment and facilities at the Dublin Institute of Technology. Thanks are due to Dr. Donagh O'Mahony and Dr. Richard Winfield for allowing the authors to use their Bentham PVE300 facility at Tyndall National Institute as well as for their assistance and helpful discussion.

\section{References}

[1] H. J. Hovel, R. T. Hodgson, and J. M. Woodall, "The effect of fluorescent wavelength shifting on solar cell spectral response," Solar Energy Materials, vol. 2, no. 1, pp. 19-29, 1979.

[2] C. Strümpel, M. McCann, G. Beaucarne et al., "Modifying the solar spectrum to enhance silicon solar cell efficiency-an overview of available materials," Solar Energy Materials \& Solar Cells, vol. 91, no. 4, pp. 238-249, 2007.

[3] E. Klampaftis, D. Ross, K. R. McIntosh, and B. S. Richards, "Enhancing the performance of solar cells via luminescent down-shifting of the incident spectrum: a review," Solar Energy Materials \& Solar Cells, vol. 93, no. 8, pp. 1182-1194, 2009.

[4] K. R. McIntosh, G. Lau, J. N. Cotsell et al., "Increase in external quantum efficiency of encapsulated silicon solar cells from a luminescent down-shifting layer," Progress in Photovoltaics: Research and Applications, vol. 17, no. 3, pp. 191-197, 2009.

[5] R. Rothemund, S. Kreuzer, T. Umundum, G. Meinhardt, T. Fromherz, and W. Jantsch, "External quantum efficiency analysis of Si solar cells with II-VI nanocrystal luminescent downshifting layers," Energy Procedia, vol. 10, pp. 83-87, 2011.

[6] D. Ross, D. Alonso-Álvarez, E. Klampaftis et al., "The impact of luminescent down shifting on the performance of CdTe photovoltaics: impact of the module vintage," IEEE Journal of Photovoltaics, vol. 4, no. 1, pp. 457-464, 2014.

[7] D. Alonso-Álvarez, D. Ross, E. Klampaftis et al., "Luminescent down-shifting experiment and modelling with multiple photovoltaic technologies," Progress in Photovoltaics: Research and Applications, vol. 23, no. 4, pp. 479-497, 2015.

[8] E. Klampaftis and B. S. Richards, "Improvement in multicrystalline silicon solar cell efficiency via addition of luminescent material to EVA encapsulation layer," Progress in Photovoltaics: Research and Applications, vol. 19, no. 3, pp. 345-351, 2011.

[9] E. Klampaftis, D. Ross, S. Seyrling, A. N. Tiwari, and B. S. Richards, "Increase in short-wavelength response of encapsulated CIGS devices by doping the encapsulation layer with luminescent material," Solar Energy Materials \& Solar Cells, vol. 101, pp. 62-67, 2012.

[10] H. Ahmed, M. Kennedy, J. Doran et al., "Lumogen violet dye as luminescent down-shifting layer for c-silicon solar cells," in Proceedings of the 27th European Photovoltaic Solar Energy Conference and Exhibition, pp. 311-313, Frankfurt, Germany, 2012.

[11] H. Ahmed, M. Kennedy, J. Doran et al., "Characterization of luminescent down-shifting materials and applications for enhanced photovoltaic devices," in Proceedings of the 8th Photovoltaic Science Application and Technology (PVSAT-8) Conference and Exhibition, pp. 1-4, Northumbria University, 2012. 
[12] H. A. Ahmed, J. Walshe, M. Kennedy, T. Confrey, J. Doran, and S. McCormack, "Enhancement in solar cell efficiency by luminescent down-shifting layers," Advances in Energy Research, vol. 1, no. 2, pp. 117-126, 2013.

[13] M. Kennedy, H. Ahmed, J. Doran et al., "Large stokes shift downshifting Eu(III) films as efficiency enhancing UV blocking layers for dye sensitized solar cells," Physica Status Solidi A: Applications and Materials Science, vol. 212, no. 1, pp. 203-210, 2015.

[14] T.-S. Ahn, R. O. Al-Kaysi, A. M. Müller, K. M. Wentz, and C. J. Bardeen, "Self-absorption correction for solid-state photoluminescence quantum yields obtained from integrating sphere measurements," Review of Scientific Instruments, vol. 78, no. 8, Article ID 086105, 2007.

[15] L. R. Wilson and B. S. Richards, "Measurement method for photoluminescent quantum yields of fluorescent organic dyes in polymethyl methacrylate for luminescent solar concentrators," Applied Optics, vol. 48, no. 2, pp. 212-220, 2009.

[16] C. Hu and R. M. White, Solar Cells: From Basic to Advanced Systems, McGraw-Hill, New York, NY, USA, 1983.

[17] R. Rothemund, "Optical modelling of the external quantum efficiency of solar cells with luminescent down-shifting layers," Solar Energy Materials and Solar Cells, vol. 120, pp. 616-621, 2014.

[18] J. Liu, K. Wang, W. Zheng, W. Huang, C.-H. Li, and X.-Z. You, "Improving spectral response of monocrystalline silicon photovoltaic modules using high efficient luminescent downshifting Eu ${ }^{3+}$ complexes," Progress in Photovoltaics: Research and Applications, vol. 21, no. 4, pp. 668-675, 2013.

[19] T. Maruyama and R. Kitamura, "Transformations of the wavelength of the light incident upon solar cells," Solar Energy Materials and Solar Cells, vol. 69, no. 3, pp. 207-216, 2001.

[20] C. De Mello Donegá, S. G. Hickey, S. F. Wuister, D. Vanmaekelbergh, and A. Meijerink, "Single-step synthesis to control the photoluminescence quantum yield and size dispersion of CdSe nanocrystals," The Journal of Physical Chemistry B, vol. 107, no. 2, pp. 489-496, 2003.

[21] D. Ross, E. Klampaftis, J. Fritsche, M. Bauer, and B. S. Richards, "Increased short-circuit current density of production line CdTe mini-module through luminescent down-shifting," Solar Energy Materials \& Solar Cells, vol. 103, pp. 11-16, 2012. 

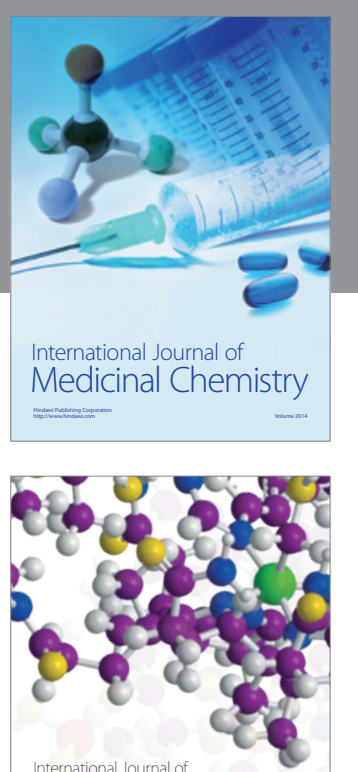

Carbohydrate Chemistry

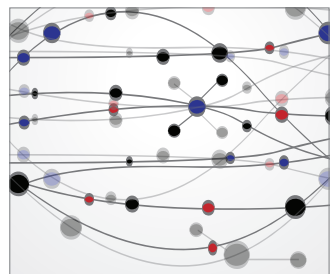

The Scientific World Journal
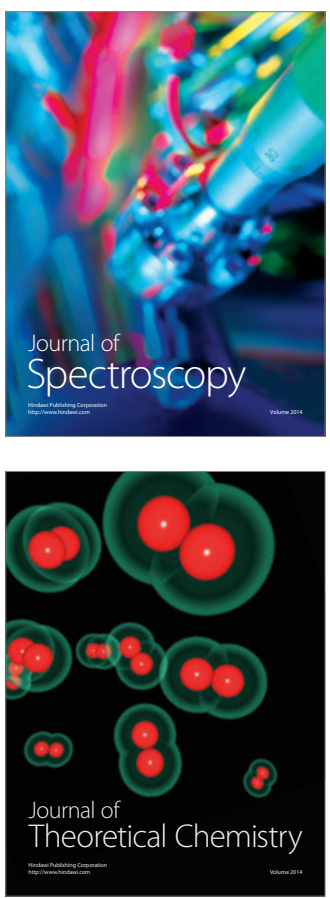
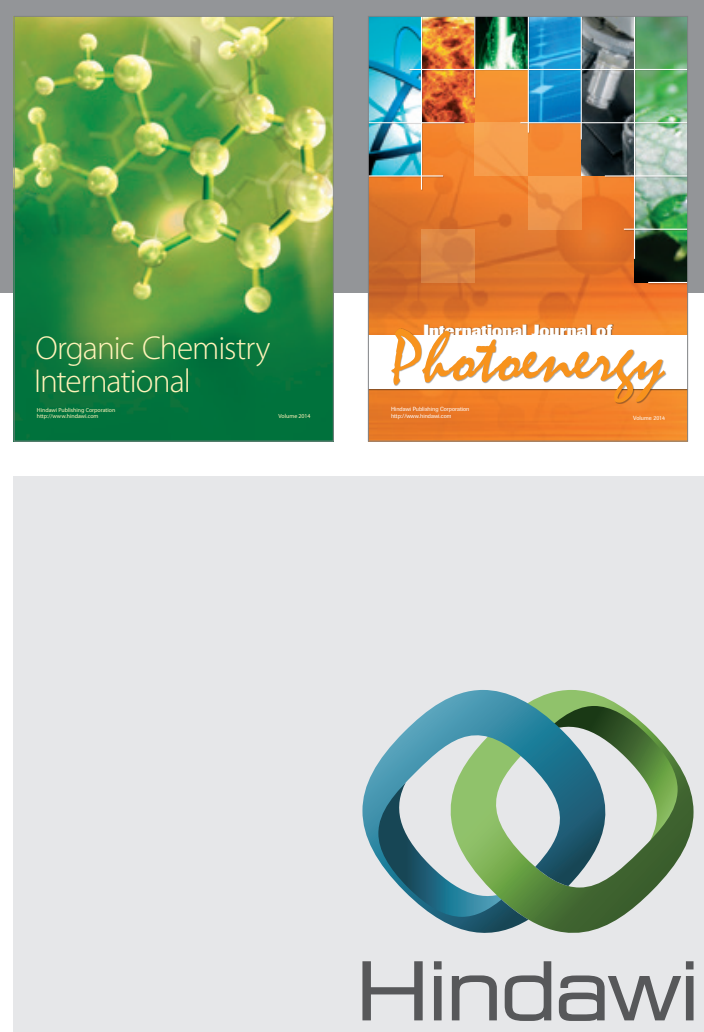

Submit your manuscripts at

http://www.hindawi.com

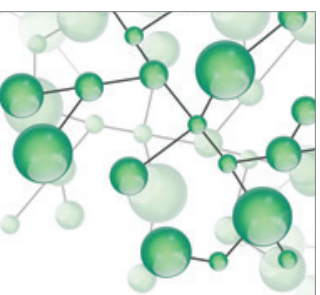

International Journal of

Inorganic Chemistry

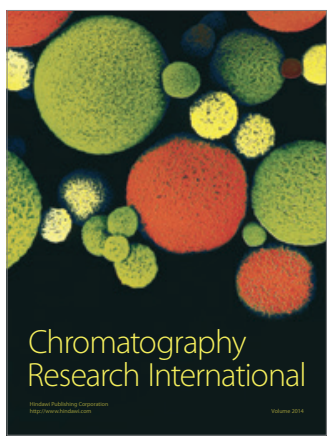

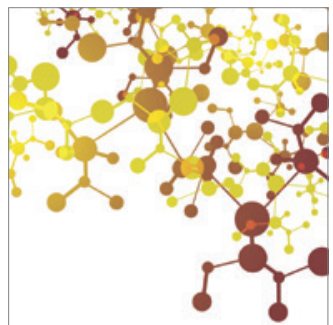

Applied Chemistry
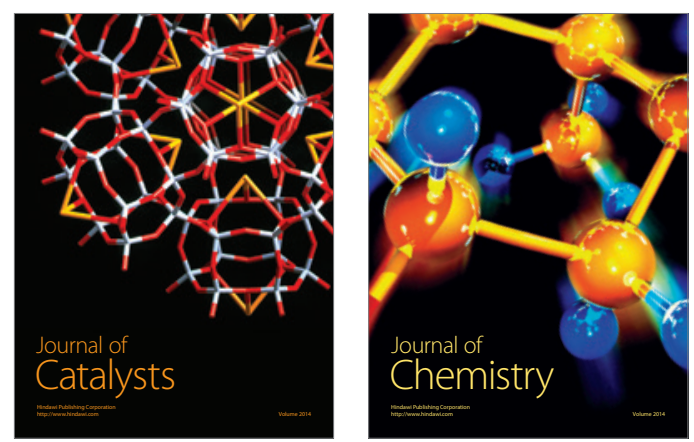
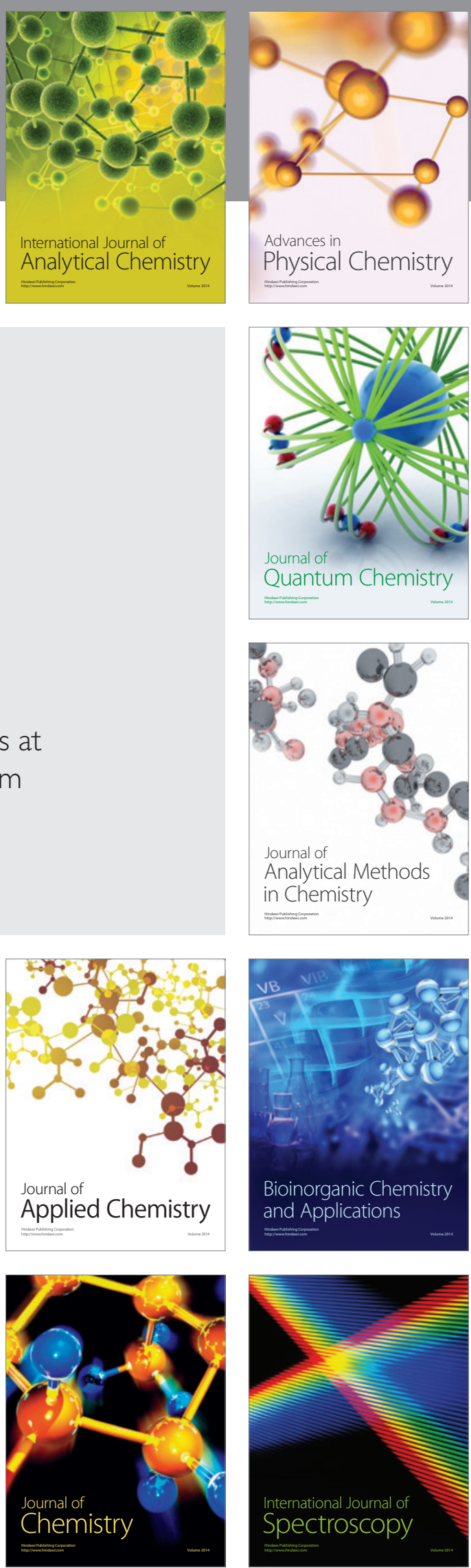